\title{
Surface Micromachined Polyimide Scanning Thermocouple Probes
}

\author{
Mo-Huang Li, Julius J. Wu, and Yogesh B. Gianchandani, Member, IEEE
}

\begin{abstract}
This paper describes micromachined scanning thermocouple probes that exploit the low thermal conductivity and the high mechanical flexibility of polyimide as a structural material. They are surface micromachined using a low-temperature six-mask process suitable for appending to a CMOS fabrication sequence. The probes are $200-1000-\mu \mathrm{m}$ long, 40-120- $\mu \mathrm{m}$ wide, and of varying thickness. They are assembled by a flip-over approach that eliminates the need for dissolving the substrate wafer or removing the probe from it. Temperature sensing is provided by thin-film $\mathrm{Ni} / \mathrm{W}$ or chromel/alumel thermopiles embedded in the polyimide, which provide Seebeck coefficients of 22.5 and $37.5 \mu \mathrm{V} / \mathrm{K}$ per junction, respectively. Modeling results indicate that the low thermal conductivity of polyimide, causes the temperature drop along the probe length to be much higher than with other candidate materials such as $\mathrm{Si}$ or $\mathrm{SiO}_{2}$, which contributes to improved thermal isolation of the sample and higher temperature sensitivity of the probe. However, the response time of the probe is compromised, and the measured $-3 \mathrm{~dB}$ bandwidth of the probes is $\approx 500 \mathrm{~Hz}$. A sample scan is presented. [561]
\end{abstract}

Index Terms-Polymide, scanning microscopy, surface micromachining, thermocouple.

\section{INTRODUCTION}

$\mathbf{T}$ HERMAL probes are of increasing interest in a variety of applications, including high resolution temperature mapping (e.g., for ULSI diagnostics), topographical mapping, photothermal absorption spectroscopy, and subsurface imaging [1]-[3]. Two applications of relevance to photolithography research are mapping of the latent image in exposed but undeveloped photoresist, and micro-calorimetric analysis of sub-micron features of resist to determine the impact of spatial boundary conditions [4], [5]. Thermal probes based on various temperature sensing techniques have been reported in the past. These include the (Wollaston) wire bolometer, the thin-film bolometer [6], the coated wire thermocouple (TC) [7], micromachined thin-film TCs [8]-[11], Schottky diodes [12], and Joule expansion [13]. The Wollaston probe has a tip diameter in the range of 5-50 $\mu \mathrm{m}$, whereas most of the other options offer tip diameters of $10 \mathrm{~nm}$ to $1 \mu \mathrm{m}$. Due to the high thermal conductivity of metals, wire-based probes may present a greater thermal load to the samples than micromachined

Manuscript received April 6, 2000; revised August 30, 2000. This work was supported in part by the Semiconductor Research Corporation under Contract 98-LP-452.005, and in part by the the Center for Nano Technology, University of Wisconsin, Madison, which is supported in part by DARPA/ONR under Grant N00014-97-1-0460. Subject Editor, G. B. Hocker.

M.-H. Li and Y. B. Gianchandani are with the Center for Nano Technology, Department of Electrical and Computer Engineering, University of Wisconsin, Madison, WI 53706-1691 USA (e-mail: yogesh@engr.wisc.edu).

J. J. Wu is with the KLA Tencor Corporation, San Jose, CA 95134 USA.

Publisher Item Identifier S 1057-7157(01)01591-8. probes fabricated from other materials. Micromachined probes that use thin film TCs are attractive because the fabrication is relatively simple, the temperature sensing can be localized, and the readout does not require a bias current that could cause spurious heating.

This effort describes a new type of TC-based thermal probe for scanning microscopy applications that is fabricated with a low-temperature, six-mask process intended for ease of integration with CMOS circuitry [14]. The probe has a built-in scanning tip that is exposed by a flip-over assembly step at the end of the processing sequence. The probe design allows it to be used for tunneling and force microscopy. In the following text, Section II presents the structure and operation of the probe, Section III describes the fabrication sequence, and Section IV describes experimental results, including sample scans and response speed measurements.

\section{STRUCTURE AND OPERATION}

The structure of the polyimide-based thermal probe is illustrated in Fig. 1. A cantilever with a thin-film TC sandwiched between two layers of polyimide is partially released from the surface of the substrate wafer and flipped over to expose the scanning tip which is initially pointing downwards. The TC has one junction located within the scanning tip, which is at the distal end of the cantilever, while the other junction is located on an unreleased region at its base. The TC may be replaced by a thermopile if necessary. The advantage of having a differential measurement from these two junctions, spaced apart only a few hundred microns, is that common mode errors resulting from global temperature drifts or other effects are automatically cancelled out. The probe incorporates a thin film resistor to provide a temperature bias with respect to the sample. In this effort the resistor is located at the base of the probe, although in general it may be located at any point along its length. The temperature bias may also be provided by other means, such as laser heating.

Scanning probes generally require the scanning tip to overhang the edge of the substrate to permit the visual alignment of the sample [15], [16]. In devices such as scanning force microscopes that use a laser pick-off, this clearance is even more important. The flip-over method for providing the tip clearance obviates the removal of the substrate material from underneath the tip, which has been performed in the past but is not favored in the presence of on-chip circuitry because of the difficulty in protecting the electronics while the substrate is being etched. Another possibility is to fabricate the probe pointing upwards on the substrate wafer [11]. In this case, the options for providing an optical path are once again to dissolve the substrate wafer 


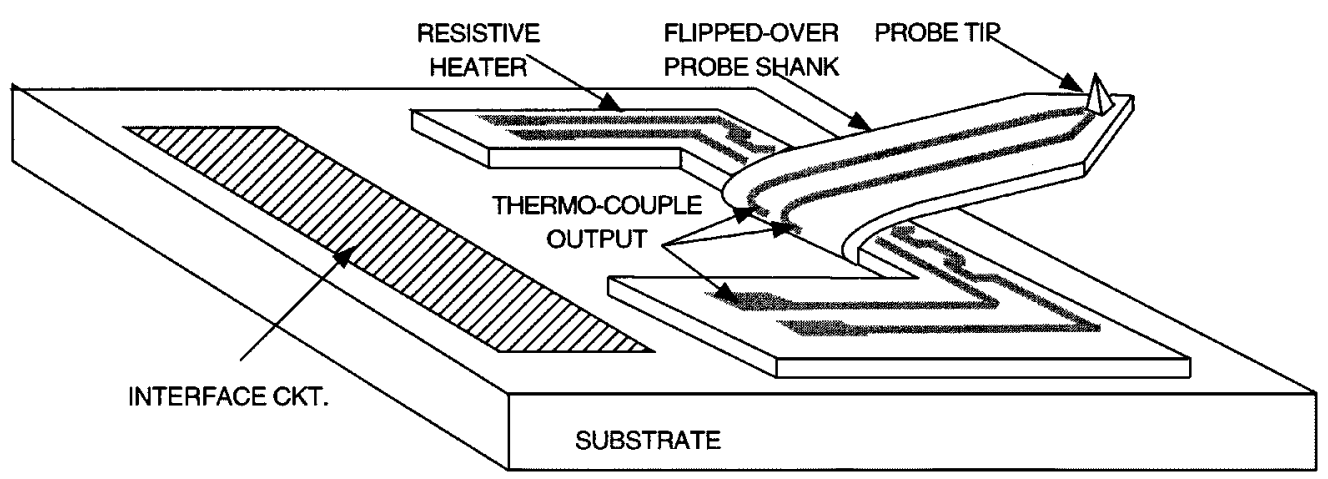

Fig. 1. Schematic of the thermal probe illustrating the flip-over approach for tip clearance.

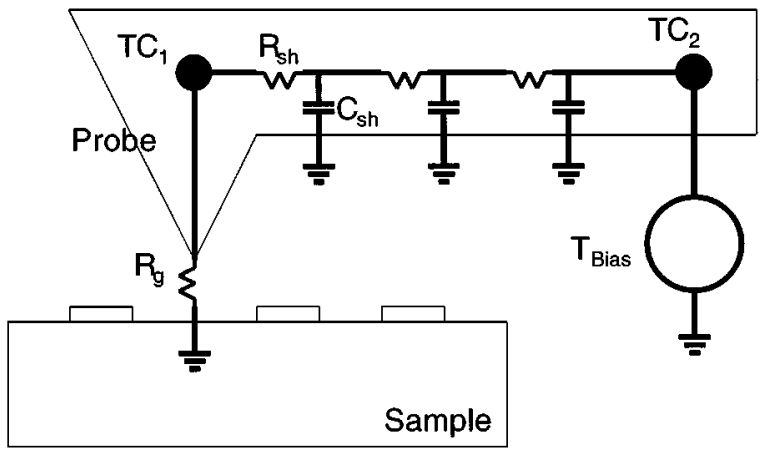

Fig. 2. Thermal conduction model for simulations. $R_{\mathrm{sh}}$ and $C_{\mathrm{sl}}$ represent distributed thermal elements that are composites of structural and TC materials of the probe. In contact mode operation, $R_{\mathrm{g}}$ is the contact and spreading (thermal) resistance.

near the probe head, or to remove the probe from the substrate entirely and relocate is elsewhere. Neither of these are attractive for an integrated solution.

The thermal probe may be operated in either contact mode, in which the tip is touching the sample, or in noncontact mode. The principle of operation is illustrated in Fig. 2. A temperature bias between the proximal TC junction and the sample causes thermal conduction across the air gap and along the probe shank. Due to the large thermal resistance of the air gap, a significant fraction of the temperature bias may be dropped across it. The remainder that is dropped along the probe shank between the two TC junctions is responsible for the readout signal. In noncontact mode operation, the thermal probe can be used to map topography because the readout signal responds to variations in the air gap. The large thermal resistances of the air gap and the probe shank minimize the thermal loading imposed by the probe upon the sample, despite any temperature bias that may exist. The contact mode operation can be evaluated as a special case of noncontact operation in which the thermal resistance between the tip and the sample is relatively small and does not track the topography. In this case, the TC signal maps variations in the surface temperature and thermal conductivity. Models for interaction between the scanning tip and sample are the subject of active research. However, much of the effort appears to be directed toward bolometer probes [2], [9], [17], [18].

A comparison of probes with different dimensions and structural materials can be made by the simple equivalent circuit model illustrated in Fig. 2. In this, $R_{\mathrm{sh}}$ and $C_{\mathrm{sh}}$ represent the dis-
TABLE I

Material Properties and Simulations Results of Thermal Probes With SI, $\mathrm{SiO}_{2}$, AND Polyimide Shanks. $T_{\mathrm{TC}}$ Is the DC PerCentage DroP of $T_{\text {Bias }}$ Along the Probe Length, While $t_{\mathrm{r}}$ Is the 10\%-90\% Rise Time OF THE TEMPERATURE RESPONSE TO A STEP CHANGE IN $T_{\text {Bias }}$

\begin{tabular}{c|c|c|c}
\hline Parameter & $\mathrm{Si}$ & $\mathrm{SiO}_{2}$ & PI 2610 \\
\hline $\mathrm{T}_{\mathrm{TC}}(\%)$ & 9.7 & 75.4 & 81 \\
\hline $\mathrm{t}_{\mathrm{r}}(\mathrm{ms})$ & 0.44 & 1.2 & 2.26 \\
\hline Th. Cond. $(\mathrm{W} / \mathrm{mK})$ & 141.2 & 1.4 & 0.147 \\
\hline Sp. Heat Cap. $(\mathrm{J} / \mathrm{gm}-\mathrm{K})$ & 0.7 & 1.4 & 1.09 \\
\hline Density $(\mathrm{gm} / \mathrm{cc})$ & 2.33 & 2.19 & 1.4 \\
\hline
\end{tabular}

tributed thermal resistance and capacitance of the probe shank, and include the structural material as well as the embedded thermocouple materials. The resistance of the air gap is labeled $R_{\mathrm{g}}$. Its lower limit, which is relevant only in contact mode, is constrained by the effective contact and spreading (thermal) resistance at the probe tip. In noncontact mode, it can be shown that for a given $R_{\mathrm{g}}$, the temperature drop along the probe length is highest when $R_{\mathrm{sh}}=R_{\mathrm{g}}[10]$. Since $R_{\mathrm{g}}$ generally exceeds $10^{\tilde{5}} \mathrm{~kW}$ and varies with operating conditions, the design guideline is to maximize $R_{\mathrm{sh}}$. The response speed of the probe is increased by reducing the product $R_{\mathrm{sh}} \mathrm{C}_{\mathrm{sh}}$. In order to optimize these two performance criteria, the structural material of the probe should have the lowest thermal conductivity and the specific heat capacity for the dimensions used. Polyimide offers an important advantage over other structural materials in this respect.

Although polyimide is not always the best choice for a structural material because it tends to absorb moisture, it has been used successfully for microstructures in the past [19]. Its use in the scanning thermal probes is worth evaluating because these devices are generally used in controlled environmental conditions, and because small variations in absorbed moisture do not directly affect their performance.

The simulated responses of $\mathrm{Si}, \mathrm{SiO}_{2}$, and polyimide shank probes are compared in Table I. The probes dimensions assumed were $200 \mu \mathrm{m}$ in length and $40 \mu \mathrm{m}$ in width, with $3-\mu \mathrm{m}$ thickness for the polysilicon and polyimide probes, and $0.5-\mu \mathrm{m}$ thickness for the oxide probe. The TC was assumed to be comprised of one $10-\mu \mathrm{m}$ wide, $2000-\AA$ thick trace of $\mathrm{Ni}$, and another of $\mathrm{W}$. The thermal resistance $R_{\mathrm{g}}$ was $120000 \mathrm{~kW}$ in each case. In response to a step change in the temperature difference between the proximal TC junction and the sample, (represented in Fig. 2 by $T_{\text {Bias }}$ ), the final temperature drop achieved across the ther- 
(a)

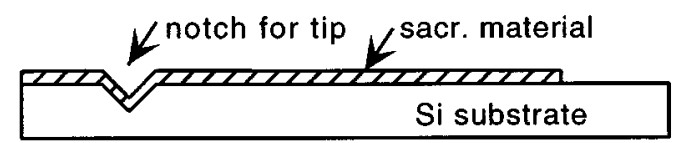

(b)

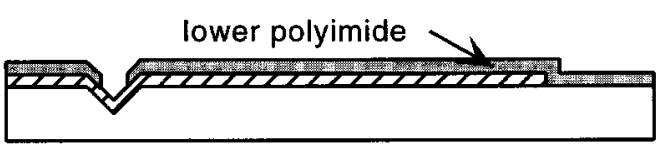

(c)

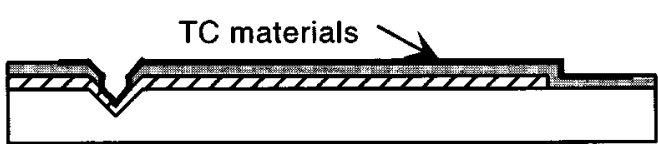

(d)

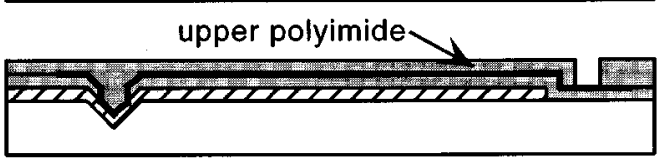

(e)

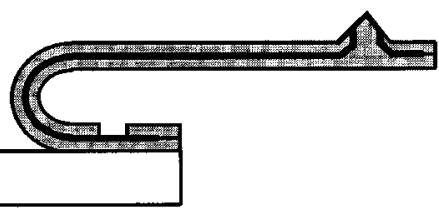

Fig. 3. Fabrication sequence: (a) Tip notch is etched into silicon wafer and sacrificial layer is deposited and patterned (masks 1, 2); (b) First polyimide is deposited and patterned, removing it from the field region and within the tip notch (mask 3); (c) TC materials are deposited and patterned (masks 4, 5); (d) Second polyimide is deposited and patterned (mask 6); (e) Sacrificial layer is etched, and the probe is flipped over and clamped in new position. Process variations are described in the text.

mocouple $\left(T_{\mathrm{TC}}\right)$ was determined, along with its $10 \%$ to $90 \%$ rise time $\left(t_{\mathrm{r}}\right)$. The results show that the polyimide probe offers almost one order of magnitude larger temperature drop along its length than the Si probe. In fact, it is also larger than the temperature drop along the oxide probe, even though the oxide probe is much thinner. However, the increased thermal resistance of the polyimide probe also results in a slower response, which is reduced by a factor of five compared to the Si probe. For many applications, this is a suitable compromise.

\section{FABRICATION}

The fabrication process uses six masks and requires (100) oriented Si substrate wafers (Fig. 3). An oxide masked anisotropic wet $\mathrm{KOH}$ etch is first used to define a pyramidal notch into which the tip will be molded. The opening is sized to allow a self-terminating etch that is 5-6- $\mu \mathrm{m}$ deep. This is designed to prevent interference from the overhang of the cantilever past the tip while scanning. A $2-\mu \mathrm{m}$ thick sacrificial layer of $\mathrm{Ti}$ is deposited and patterned using a photoresist masked wet etch in dilute $\mathrm{HF}$. The $\mathrm{Ti}$ is removed only from the areas where the probe will be anchored to the substrate. The first polyimide layer is then spun on, cured, and patterned, removing it from the field regions and from within the notch. DuPont's Pyralin PI2610 is suitable because its coefficient of thermal expansion $(3 \mathrm{ppm} / \mathrm{K})$ is close to that of $\mathrm{Si}$, which minimizes the curl in the probe shank when it is eventually released from the substrate. An $\mathrm{Al}$ hard mask is used with a dry reactive ion etch of $\mathrm{O}_{2}$ and $\mathrm{SF}_{6}$ to pattern the polyimide. The TC metals are deposited and patterned in the following two masking steps. Both Ni/W and

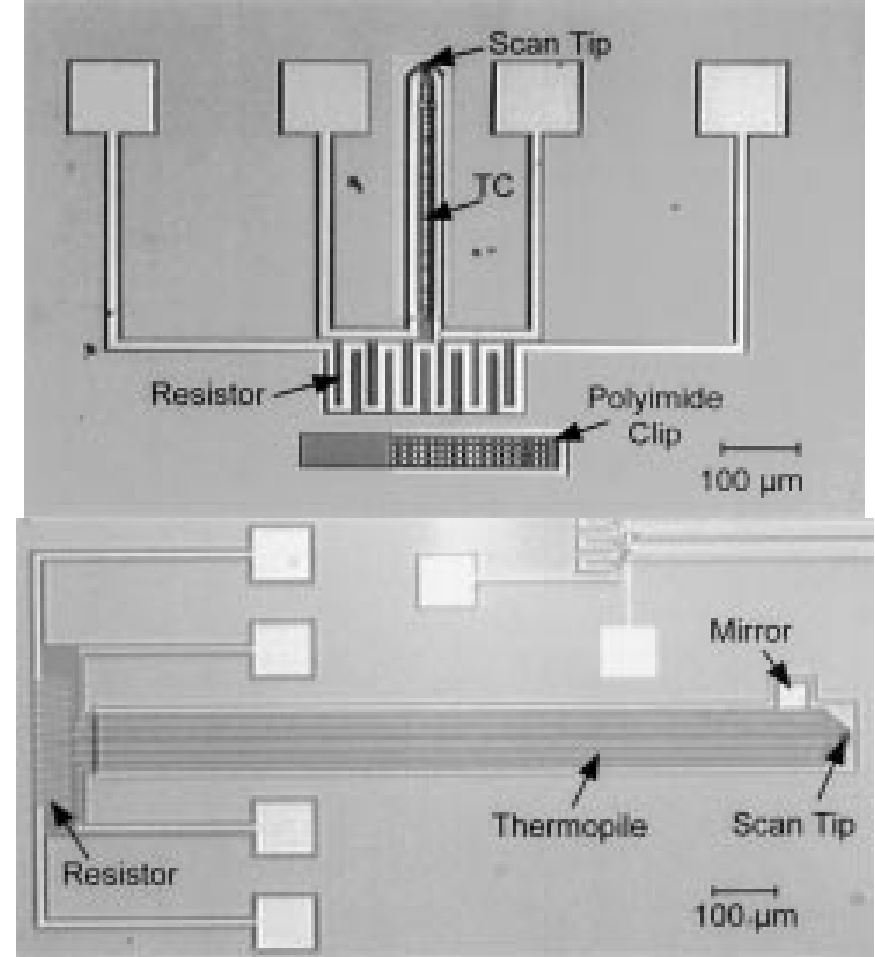

Fig. 4. Optical micrographs of fabricated thermal probes immediately before release.

chromel/alumel TCs have been investigated. One of the metals (Ni in the former case, and chromel in the latter) also forms the integrated heating resistor. Note that $\mathrm{Ni}$ may be replaced by $\mathrm{Au}$ for tunneling microscopy applications. Next, the second polyimide layer is spun on, cured and patterned by a dry etch. This etch terminates on the sacrificial $\mathrm{Ti}$ in the field and on the metal TC pads on the probe. Finally, the probe is released, manually flipped over, and restrained with an integrated polyimide clip or with a dab of epoxy. The polyimide shank is flexible and robust - no cracking or breakage has been observed in the many tens of samples that have been assembled in this manner. (In the future, a the stress mismatch may be intentionally introduced between the two polyimide layers to selectively curl portions of the probe shank after release [19]. This may help to automate the flip-over assembly. Another option may be the use of ultrasonic actuation [20].) The fabrication process has a very low thermal budget. Once the oxide mask for the $\mathrm{KOH}$ has been deposited in the first step of this process, the highest temperature encountered is the $350^{\circ} \mathrm{C}$ cure for polyimide. Unreleased probes are shown in Fig. 4, and released probes before and after they are flipped over are shown in Fig. 5.

The diameter of the scanning tip is determined by the sharpness of the notch within which it is formed. The self-terminating anisotropic wet etch provides a tip diameter of $100-200 \mathrm{~nm}$, as shown in Fig. 6. Although this is adequate for many applications, it can be reduced to about $10 \mathrm{~nm}$ by thermally oxidizing the notch prior to depositing the sacrificial Ti [8].

The use of polyimide as the structural material permits the thickness of the probe shank to be scaled in the range of 1-20 $\mu \mathrm{m}$ as a variable for adjusting the spring constant of the cantilever. For several of the devices reported here, each of the two 


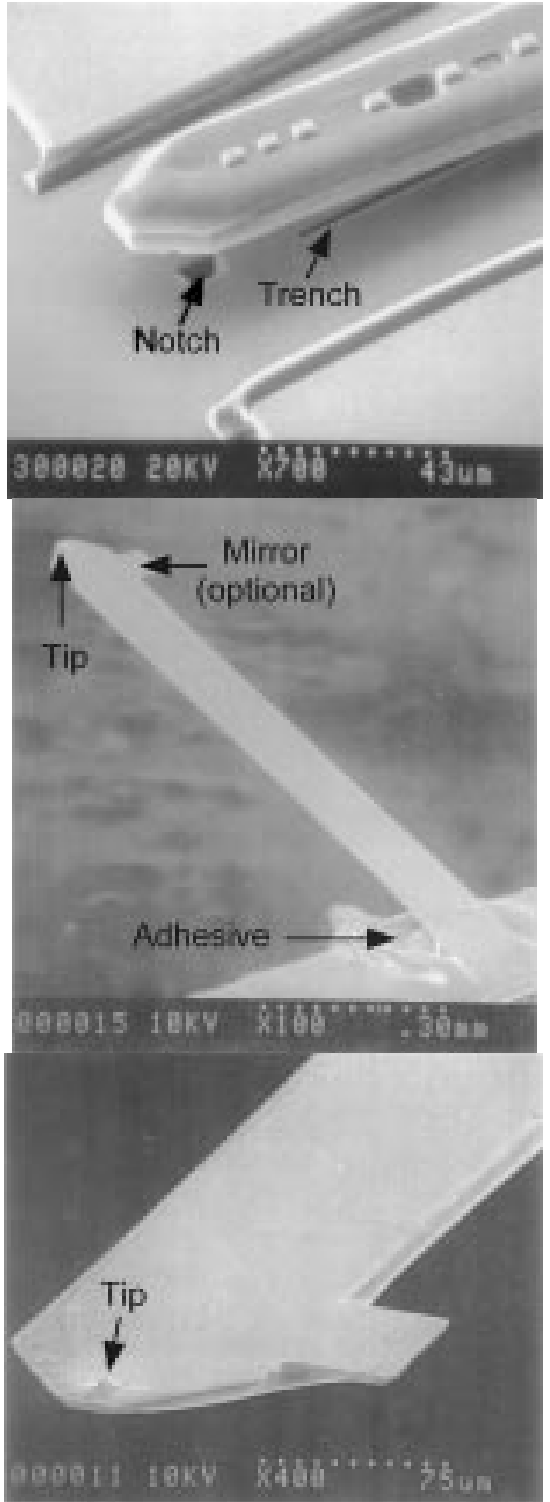

Fig. 5. (a: top -c: bottom) SEM images of (a) A scanning probe tip, immediately after release from the mold; (b) An $800 \mu \mathrm{m}$ long probe flipped over the die edge and held down with epoxy; (c) A close-up of the probe shank, showing the scan tip and the paddle-shaped mirror.

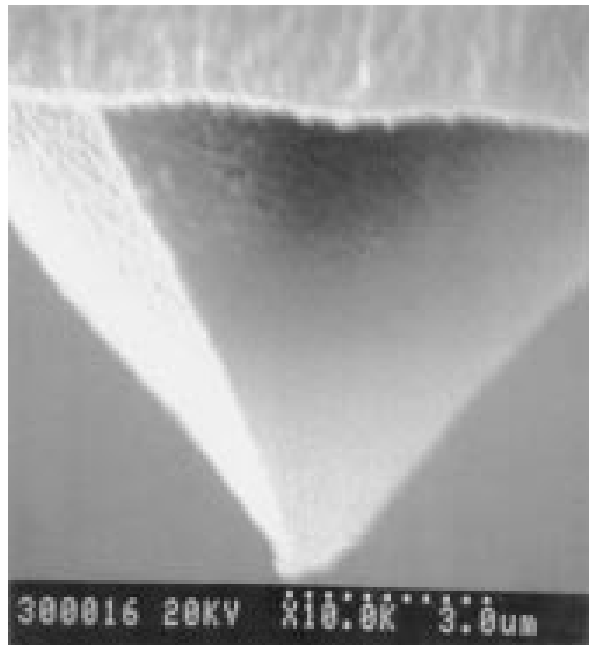

Fig. 6. A close-up of the tip formed without oxide-sharpening the mold.

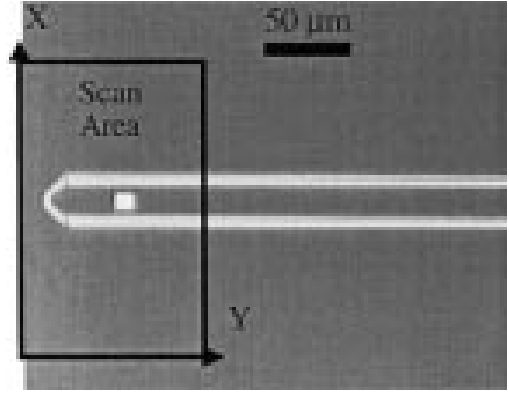

Fig. 7. Optical micrograph of the scan sample used in Fig. 8, showing a $263 \Omega$ metal resistor patterned on top of $4 \mu \mathrm{m}$ polyimide on a Si substrate. A DC current of $4 \mathrm{~mA}$ was applied to the resistor.

polyimide layers was $\approx 1.55-\mu \mathrm{m}$ thick PI2610. For a $200-\mu \mathrm{m}$ long, $40-\mu \mathrm{m}$ wide probe, this yields a spring constant of 0.31 $\mathrm{N} / \mathrm{m}$, whereas for a $1000-\mu \mathrm{m}$ long, $120-\mu \mathrm{m}$ wide probe, it yields $7.4 \times 10^{-3} \mathrm{~N} / \mathrm{m}$. Another option for adjusting the shank stiffness is to mold a longitudinal rib on its underside. This can be done without any additional processing steps by etching a narrow trench along the length of the shank during the formation of the notch for the scan tip. Making the trench narrower than the notch ensures that it is also shallower than the notch, so the polyimide rib molded by it does not protrude higher than the scan tip once the probe is flipped over, where it might otherwise interfere with a scan. A close-up of the trench and the notch underneath a released probe are shown in Fig. 5(a). Another process variation that can be used to tailor the mechanical stiffness and the thermal conduction along the probe without increasing the mask count is to not strip the metal that serves as the hard mask for the dry etch of the second polyimide layer.

\section{MEASUREMENT Results}

As mentioned in the preceding section, both $\mathrm{Ni} / \mathrm{W}$ and chromel/alumel TCs have been investigated. The resistive heater located at the base of each probe permits in-situ testing of the TCs [10]. The TC output is monitored while the junction near the probe tip is held at room temperature and the junction at the base is heated by the resistor. However, it should be noted that due to the low thermal conductivity of the polyimide there can be a significant temperature drop between the resistor and the heated junction. Accounting for this by finite element modeling, the experimentally measured values of Seebeck coefficient for $\mathrm{Ni} / \mathrm{W}$ and chromel/alumel junctions are 22.5 and $37.5 \mu \mathrm{V} / \mathrm{K}$, respectively. These compare well with predictions based on published values of the thermo-electric power of these materials, which are 25.6 and $41.0 \mu \mathrm{V} / \mathrm{K}$, respectively. The measured temperature coefficient of resistance (TCR) of the $\mathrm{Ni}$ and chromel thin films that constitute the resistor is 4282 $\mathrm{ppm} / \mathrm{K}$ and $435 \mathrm{ppm} / \mathrm{K}$, respectively.

A fabricated polyimide probe with a Ni/W TC was used in the contact mode scan of the sample in Fig. 7. The scan direction was parallel to the $x$-axis as indicated in the figure. The scanned area was a portion of a $263 \Omega$ metal resistor patterned on a $\mathrm{Si}$ substrate and isolated from it by a $4-\mu \mathrm{m}$ thick layer of polyimide. A dc of $4 \mathrm{~mA}$ was passed through the resistor. The thermopile output, presented in Fig. 8, shows that the temperature peak is located near the apex formed by the bend between the 


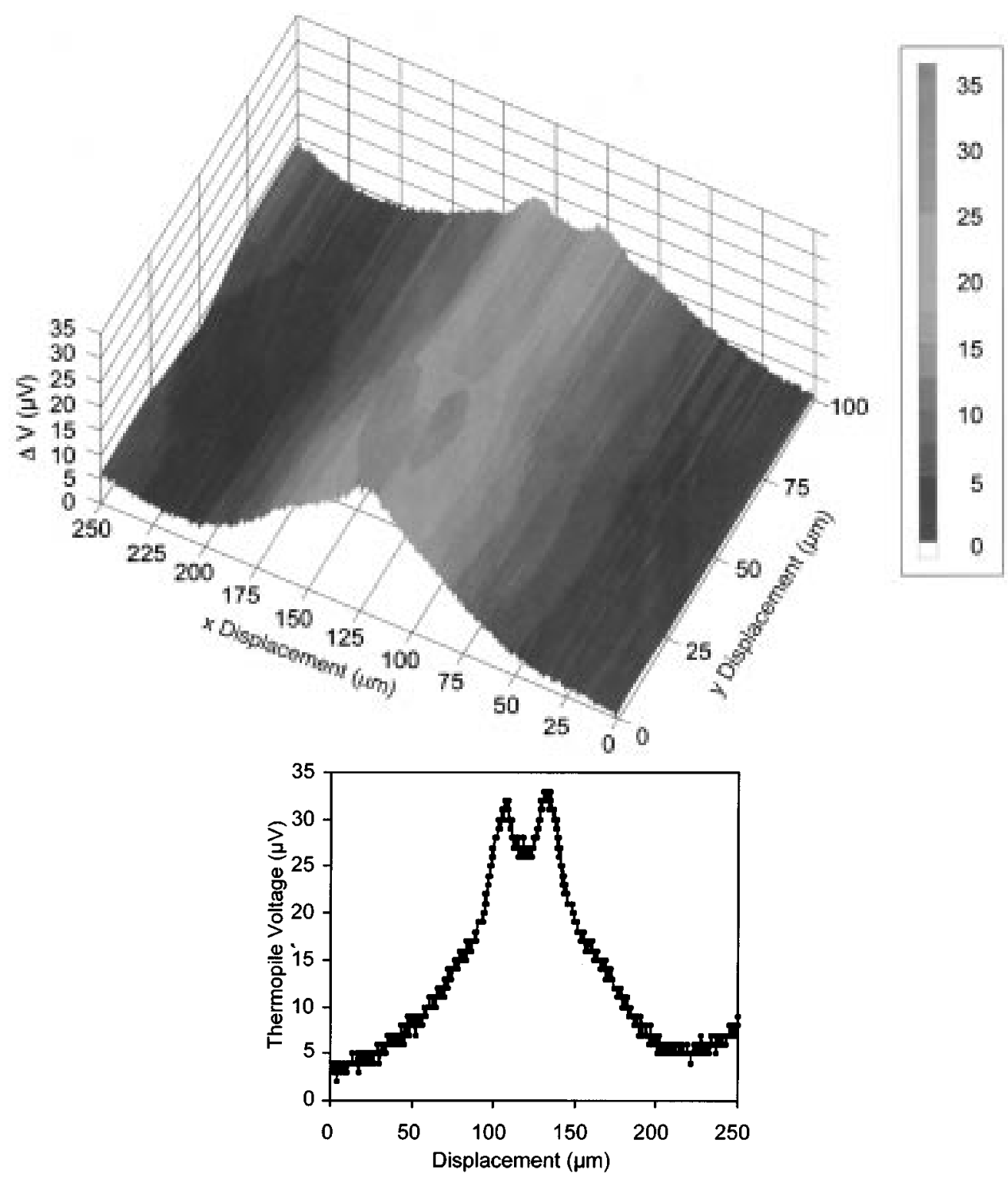

Fig. 8. (a) The scanned thermal image of the resistor in Fig. 7 with the thermopile response on the vertical axis. The scan clearly shows that the apex of the sample resistor is hotter than the surrounding areas. (b) A linear scan from the same sample.

two linear segments of the resistor. These segments are $10 \mu \mathrm{m}$ wide and $20 \mu \mathrm{m}$ apart where they run parallel to each other.

The response speed or bandwidth of the thermal probe can be determined by a measurement similar to the one used for TC characterization. In this, a sinusoidal voltage is applied to the integrated heater while the scan tip is held at room temperature. Since the input power is proportional to the square of the applied voltage, a sinusoid at frequency $f$ centered at ground (without a DC bias) generates a power signal at $2 f$, and consequently a TC output at $2 f$. The presence of a DC bias causes the power signal to have components at both $f$ and $2 f$, and must be avoided. Some capacitive feed-through of the input signal may also occur at $f$. As the input signal frequency is increased, the TC is eventually unable to keep up with the power oscillations, and the amplitude of the output signal decreases. The measured bandwidth of two probes is shown in Fig. 9. These measurements were performed on P1, a 200- $\mu \mathrm{m}$ long, $40-\mu \mathrm{m}$ wide, 3.1- $\mu \mathrm{m}$ thick probe with a single embedded pair of TC metal lines, and

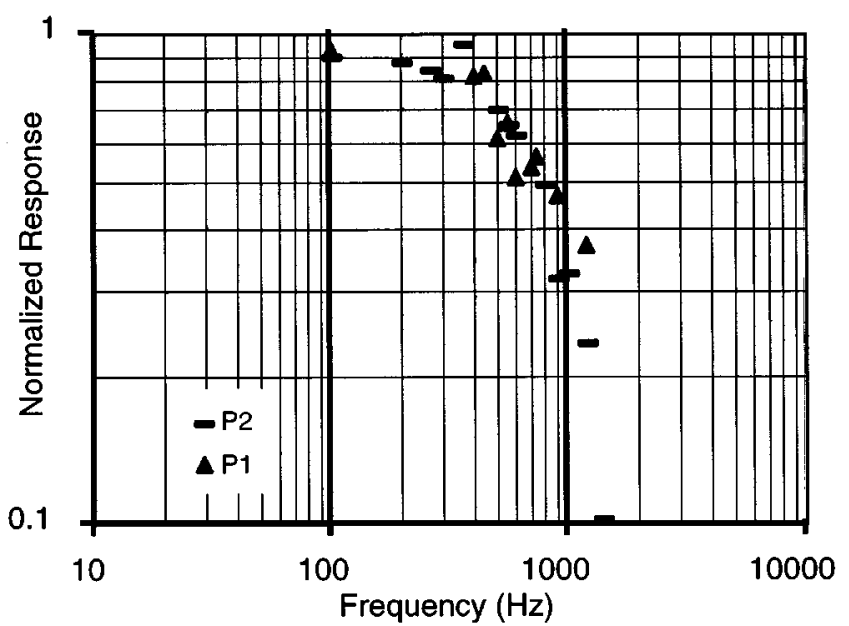

Fig. 9. Bandwidth measurements of thermocouple and thermopile probes show a corner frequency of $\approx 500 \mathrm{~Hz}$. 
P2, a 1000- $\mu \mathrm{m}$ long, $120-\mu \mathrm{m}$ wide, $3.1-\mu \mathrm{m}$ thick probe with five pairs of embedded TC metal lines. The input signals for the TC and the thermopile had 5 and $3 \mathrm{~V}$ peak-to-peak amplitudes, respectively. Both output signals showed a 3-dB bandwidth near $500 \mathrm{~Hz}$, suggesting that the scan speed should not exceed $2 \mathrm{~ms}$ per pixel, for these probes.

\section{CONCLUSION}

This effort has described polyimide-based scanning thermal profilers with integrated tips and embedded thin-film TCs for temperature sensing. The very low thermal conductivity and the very high mechanical flexibility of polyimide are exploited in these devices. Simulations suggest that using polyimide as a structural material increases the temperature drop along the probe length by about $10 \times$ compared to a Si probe of the same dimensions. The response speed is reduced by about $5 \times$, which is an acceptable compromise for many applications. The polyimide probes are surface micromachined on a Si substrate using a low-temperature six-mask process suitable for appending to a CMOS process sequence. They are assembled by flipping over the cantilever, allowing the scanning tip to overhang the edge of the chip for easy alignment to the sample and for optical access to a laser when necessary. The probes, which may be used for force and tunneling microscopy as well, are $200-1000 \mu \mathrm{m}$ long, 40-120 $\mu \mathrm{m}$ wide, and of varying thickness. Tip diameters are in the range of $200 \mathrm{~nm}$, but can be sharpened to about $10 \mathrm{~nm}$ without the use of additional lithography steps. The integrated thin film thermopiles of Ni/W and chromel/alumel offer a temperature sensitivity of about 22.5 and $37.5 \mu \mathrm{V} / \mathrm{K}$ per junction, respectively. Other materials may be used for higher sensitivity within the constraints of process compatibility. Scan results of a $263 \Omega$ wire carrying $4 \mathrm{~mA}$ are presented. Frequency response measurements of a $200-\mu \mathrm{m}$ long, single TC probe and a $1000-\mu \mathrm{m}$ long, thermopile probe both show $-3-\mathrm{dB}$ bandwidth $\approx 500 \mathrm{~Hz}$

\section{REFERENCES}

[1] H. K. Wickramasinghe, "Scanned-probe microscopes," Sci. Amer, vol. 261, no. 4, pp. 98-105, 1989.

[2] A. Hammiche, H. M. Pollock, M. Song, and D. J. Hourston, "Sub-surface imaging by scanning thermal microscopy," Meas. Sci. Technol., vol. 7, no. 2, pp. 142-50, 1996.

[3] J. Varesi, S. Muenster, and A. Majumdar, "High-resolution current and temperature mapping of electronic devices using scanning joule expansion microscopy," in IEEE 36th Аnnu. Int. Reliability Physics Symp., 1998, pp. 169-72.

[4] L. E. Ocola, D. Fryer, G. Reynolds, A. Krasnoperova, and F. Cerrina, "Scanning force microscopy measurements of latent image topography in chemically amplified resists," Appl. Phys. Lett., vol. 68, no. 5, pp. 717-19, 1996.

[5] D. Fryer, J. de Pablo, and P. Nealey, "Investigation of photoresist/wafer interface with a local thermal probe," SPIE, vol. 3333, pp. 1031-9, 1998.

[6] M. -H. Li and Y. B. Gianchandani, "Microcalorimetry applications of a surface micromachined bolometer-type thermal probe," J. Vac. Sci. Technol. B, vol. 18, no. 6, 2000.

[7] C. Williams and H. Wickramasinghe, "Scanning thermal profiler," Appl. Phys. Lett., vol. 49, no. 23, pp. 1587-9, 1986.

[8] Y. Suzuki, "Novel Microcantilever for scanning thermal imaging microscopy," Jpn. J. Appl. Phys., pt. 2, vol. 35, no. 3A, pp. L352-4, 1996.
[9] K. Luo, Z. Shi, J. Varesi, and A. Majumdar, "Sensor nanofabrication, performance, and conduction mechanisms in scanning thermal microscopy,” J. Vac. Sci. Technol. B, vol. 15, no. 2, pp. 349-60, 1997.

[10] Y. B. Gianchandani and K. Najafi, "A silicon micromachined scanning thermal profiler with integrated elements for sensing and actuation," IEEE Trans. Electron Devices, vol. 44, pp. 1857-67, Nov. 1997.

[11] G. Mills, H. Zhou, A. Midha, L. Donaldson, and J. M. R. Weaver, "Scanning thermal microscopy using batch fabricated thermocouple probes," Appl. Phys. Lett., vol. 72, no. 22, pp. 2900-2, 1998.

[12] T. Leinhos, M. Stopka, and E. Oesterschulze, "Micromachined fabrication of Si cantilevers with Schottky diodes integrated in the tip," Appl. Phys. A, vol. 66, pp. S65-9, 1998.

[13] A. Majumdar and J. Varesi, "Nanoscale temperature distributions measured by scanning joule expansion microscopy," J. Heat Transfer, vol. 120, no. 2, pp. 297-305, 1998.

[14] M. -H Li, J. Wu, and Y. B. Gianchandani, "High performance scanning thermal probe using a low-temperature polyimide-based micromachining process," in Proc. IEEE Int. Conf. Micro Electro Mechanical Systems (MEMS '00), Miyazaki, Japan, Jan. 2000, pp. 763-8.

[15] T. Akiyama, D. Lange, C. Hagleitner, A. Tonin, O. Brand, H. Baltes, U. Staufer, and N. F. de Rooij, "Active and self-detecting cantilever with on-chip CMOS electronics for scanning force microscopy," in Proc., IEEE Int. Conf. Sensors and Actuators (Transducers '99), Sendai, Japan, June 1999, pp. 1848-51.

[16] J. Thaysen, A. Boisen, O. Hansen, and S. Bouwstra, "AFM probe with piezoresistive read-out and highly symmetrical wheatstone bridge arrangement," in Proc. IEEE Int. Conf. Sensors and Actuators (Transducers '99), Sendai, Japan, June 1999, pp. 1852-5.

[17] F. Depasse, S. Gomès, N. Trannoy, and Ph. Grossel, "AC thermal microscopy: a probe-sample thermal coupling model," J. Phys. D: Appl. Phys., vol. 30, pp. 3279-85, 1997.

[18] S. Gomès, F. Depasse, and Ph. Grossel, "3D thermal wave scattering on buried inhomogeneities in ac thermal microscopy," J. Phys. D: Appl Phys., vol. 31, pp. 2377-87, 1998.

[19] M. Ataka, A. Omodaka, N. Takeshima, and H. Fujita, "Fabrication and operation of polyimide bimorph actuators for ciliary motion system," $J$. Microelectromechanical Sys., vol. 2, pp. 146-150, Dec. 1993.

[20] V. Kaajakari and A. Lal, "Pulsed ultrasonic release and assembly of micromachines," in Proc. IEEE Int. Conf. Solid-State Sensors and Actuators (Transducers '99), June 1999, pp. 212-215.

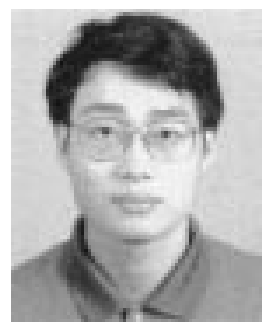

Mo-Huang Li received the B.S. and M.S. degrees from the National Sun Yet-Sen University, Koohsiung, Taiwan, and the New Jersey Institute of Technology, NJ, in 1991, and 1997 respectively. $\mathrm{He}$ is currently working toward the Ph.D. degree in electrical and computer engineering at the University of Wisconsin, Madison, WI

His research interests include all aspects of design and fabrication of micromachined sensors and their interface circuits. Currently, he is working on developing micromachined polyimide scanning thermal probes for photoresist applications.

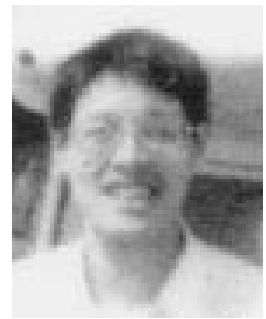

Julius Wu received the B .S. and M. S. degrees in electrical and computer engineering from the University of Wisconsin, Madison, WI.

Since 1999, he has been working as an applications development engineer for the Automatic Defect Classification group of KLA-Tencor Corporation, SanJose, CA. He is currently engaged in improving inspection systems for rapid defect detection and classification, as well as providing yield management solutions to industrial semiconductor fabrication facilities. 


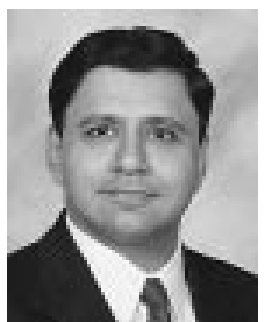

Yogesh B. Gianchandani (S'83-M'95) received the B.S. degree from the University of California, Irvine, in 1984, the M.S. degree from the University of California, Los Angeles, in 1986, and the Ph.D. degree from the University of Michigan, Ann Arbor, in 1994, all in electrical engineering.

He was with the Xerox Corporation from 1985 to 1988, and with Microchip Technology from 1988 to 1989 , working in the area of integrated circuit design. From 1994 to 1997, he was a Research Fellow at the Center for Integrated Sensors and Circuits, University of Michigan, Ann Arbor, MI. Since then, he has been an Assistant Professor in the Department of Electrical and Computer Engineering, at the University of Wisconsin, Madison, WI. He has served as a consultant to industry for the design and fabrication of a number of microelectromechanical devices. Prof. Gianchandani received the National Science Foundation Career Award in 2000. $\mathrm{He}$ has served as a Section Eeditor for the Journal of Sensors and Actuators since September 2000, and on the steering committee for the IEEE International Conference on Micro Electro Mechanical Systems (MEMS) since 1999. 